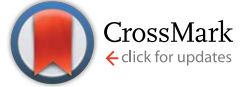

Cite this: J. Mater. Chem. C, 2015, 3, 2195

Received 1st December 2014 Accepted 22nd January 2015

DOI: $10.1039 / c 4 t c 02746 a$

www.rsc.org/MaterialsC

\section{Wiring functional groups in mesoporous organosilica materials $\uparrow$}

\begin{abstract}
M. Luka and S. Polarz
Developing future stimuli-responsive materials into more sensitive and more specific devices requires that different entities with advanced functionalities can be organized precisely, allowing them to display cooperative action. Combining the profile of properties of mesoporous materials with a large amount of organically functionalized surfaces on the one hand and conducting polymers on the other hand, for instance the polythiophene family, could lead to interesting smart materials. We show how the surface groups in periodically ordered mesoporous organosilicas (PMOs) can be electrically contacted by means of intrapore polymerization of significant amounts of poly-3,4-ethylenedioxythiophene (PEDOT). Cyclic voltammetry measurements of Ru" coordinated to the surfaces show that electrochemical charging and discharging of the material is possible. Further, the resulting, novel nanohybrid PEDOT@PMO material is capable of distinguishing between transition metal species according to the degree of Lewis-acidity. The latter was explored using impedance spectroscopy.
\end{abstract}

\section{Introduction}

Every functional material fulfils one task, as soon as it is exposed to suitable conditions. Although stimuli-responsive materials (SRMs) also belong to the large class of functional materials, they are distinct in the sense that certain properties can be activated, deactivated or at least modified depending on the prior application of a defined trigger, physical or chemical in nature. This special feature rationalizes large interest in SRMs and has stimulated to designate those materials even as smart. ${ }^{1-6}$

The earliest examples of SRMs found in the literature describe some rather simple systems such as thermoresponsive polymers, which show a change in shape because of the changing temperature. ${ }^{7}$ However, the applicability of chemically simple, one component systems is restricted concerning mastering of more demanding tasks, because up to three different functional entities need to be addressed and integrated for proper SRM-operation. The first necessary functional entity secures the detection of the initial stimulus and is attached to an information forwarding entity, until this information is processed in the response unit leading to a change of key properties of the material. Considering the diversity of potential triggers (temperature, radiation, mechanical force, pH-value, ions, redox potential, etc.) and responses (change of

Department of Chemistry, University of Konstanz, 78457 Konstanz, Germany. E-mail: sebastian.polarz@uni-konstanz.de

$\dagger$ Electronic supplementary information (ESI) available: (S1) Additional analytical data for $\mathrm{FeCl}_{3} \mathrm{UKON2a}$; (S2) additional analytical data for PEDOT@UKON-2a; (S3) additional analytical data for VO@PEDOTUKON2a; (S4) UV/Vis data for treatment of pure PEDOT with [VO](acac) ${ }_{2}$. See DOI: $10.1039 / \mathrm{c} 4 \mathrm{tc02746a}$ conductivity, optical properties, catalytic activity, wettability, etc.), it becomes clear that the design of advanced SRMs needs an architecture composed of several components assembled in a controlled fashion..$^{8}$ Furthermore, as soon as the detection of the trigger occurs via an interface, which will be the case for most chemical triggers, it is desirable to use materials with large surface areas.

Because of their large internal interfaces and the high state of development regarding surface functionalization, nanoporous solids ${ }^{9}$ appear as promising candidates as one of the components for upcoming SRMs. In particular for ordered mesoporous silica materials, there has been marked progress on the derivatization with organic groups. ${ }^{\mathbf{1 0 - 1 6}}$ Of particular relevance for the current contribution is the alternative to prepare mesoporous organosilica materials with 100\% degree of organic modification. ${ }^{17}$ The so-called PMOs (periodically ordered mesoporous organosilicas ${ }^{\mathbf{1 8}}$ are prepared using a templating approach and special sol-gel precursors with bridging organic groups like $(\mathrm{EtO})_{3} \mathrm{Si}-\mathrm{R}-\mathrm{Si}(\mathrm{OEt})_{3} \cdot{ }^{19-21} \mathrm{An}$ inspiring review about modern applications of PMO materials was published recently. ${ }^{22}$ Inagaki et al. became interested in PMO materials containing bridging phenyl $\left(\mathrm{C}_{6} \mathrm{H}_{4}\right)$ groups in $2002 .{ }^{23}$ We have introduced a related system, in which a variety of functional groups can be attached to the 3-position of the aromatic ring, the so-called UKON system. ${ }^{24}$ Meanwhile, we have presented UKON-materials with acidic groups, alkaline groups, ligand functionalities and even groups with chirality or special optical properties. ${ }^{24-33}$

For exploiting the enormous potential of such tailor-made surfaces in mesoporous organosilicas for SRMs it becomes vital, that one learns how to read out information or signals from 
these surfaces. The current contribution is one important step into this direction. Our idea addresses conducting polymers as 'wires' for electrically contacting the functional groups in the pores of PMO materials.

Conducting organic polymers represent a class of compounds of utmost value. ${ }^{34,35}$ Their electric conductivity, color or fluorescence is affected significantly by changes in the near environment. ${ }^{35-41}$ Thus, conducting have already been successfully used in SRM-related applications, for instance in photovoltaic cells, ${ }^{\mathbf{4 2}}$ as a functional material that changes its electrical properties when irradiated with light. Other applications are as thin films for organic electrodes, ${ }^{\mathbf{4 3 - 4 5}}$ in electrocatalysis or energy storage. ${ }^{\mathbf{4 6}}$ Poly(3,4-ethylenedioxythiophene) (PEDOT) is an intrinsic semiconductor and can develop high conductivities, but unlike other conducting polymers it retains substantial conductivity even in its non-doped state. ${ }^{47}$ It is also relatively stable against air, moisture and irradiation.

It is important to note some significant achievements regarding the incorporation of conducting polymers into mesoporous matrices. Bein et al. introduced polyaniline in unmodified, siliceous MCM-41 already in $1994 .^{48,49}$ The latter approach was extended significantly by Asefa et al. in 2012, who introduced roughly $4 \mathrm{wt} \%$ polyaniline inside an amine functionalized mesoporous organosilica material. ${ }^{50}$ Other examples are polymers like poly(phenylene butadiynylene) or polypyrrole in mesoporous silica. ${ }^{51,52}$ Wolf et al. published an article in 2010 in which they polymerized poly(3,4-ethylenedioxythiophene) in mesoporous silica microspheres by filling the pores with 3,4-ethylenedioxythiophene (EDOT) and adding an aqueous sodium persulfate solution. ${ }^{53-55}$ This led to a composite that consists of $50 \mathrm{wt} \%$ PEDOT and contains significant amounts of conducting polymer outside the pores. This enabled the possibility to etch the silica to obtain PEDOT microspheres. However, there is no systematic study examining the effect of functionalized pore walls interacting with an incorporated conducting polymer. Furthermore, introducing a high percentage of polymer without larger amounts of it being located outside the pores is still a difficult task. This is important, because for the composite material one is primarily interested in the interaction of the polymer with the pore-walls and not in the external polymers. Further, PEDOT is almost insoluble in any solvent and this prohibits the synthesis of the conducting polymer and its introduction into the pore-system by liquid infiltration.

Considering the high density and variability of functional groups in UKON materials, it seemed promising to us to incorporate conducting polymers (PEDOT) into the mesoporous matrix. The functional groups of the organosilica materials were selected according to their ability to coordinate to metal ions. ${ }^{28,29}$ Our task is then to find, if these metal ions coordinated to the pore-walls have an effect on the conductivity of PEDOT, and if there is any form of electronic contact. Then, it would be possible to use the materials for sensor arrays ${ }^{56-58}$ and also for electro-catalysis or for supercapacitors. ${ }^{59,60}$ Our basic idea and the targeted architecture of the hybrid materials is schematically shown in Scheme 1.

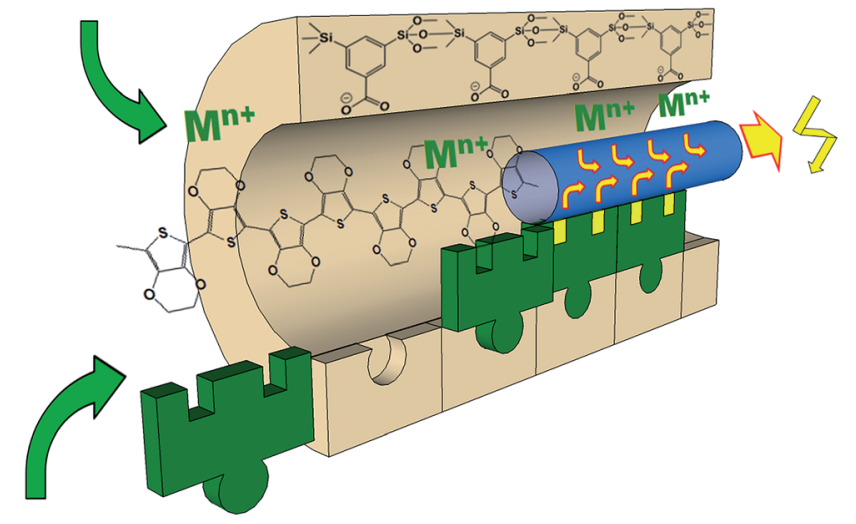

Scheme 1 One individual pore of a PMO material comprising benzoic acid in the pore walls (UKON-2a). ${ }^{24}$ The $-\mathrm{COO}^{-}$group can coordinate to various metal cations $\mathrm{M}^{n+28}$ The conducting polymer PEDOT will be prepared inside the pores and it is expected that this enables electronic communication between the pore-walls and the conducting polymer.

\section{Experimental part}

\section{Material preparation}

All reactions were performed in dried glassware in the absence of air and moisture if not indicated otherwise. All chemicals were obtained from Aldrich and dried and purified carefully. 1,3-Bis-tri-isopropoxysilylbenzoic acid was prepared as published recently. ${ }^{24}$

UKON-2a. The mesoporous organosilica material containing benzoic acid groups reported in ref. 24 was prepared by a modified procedure. Pluronic F127 (0.4 g) and bis-tri-isopropoxysilylbenzoic acid $(0.5 \mathrm{~g})$ were dissolved in $1.3 \mathrm{ml}$ of ethanol. $0.29 \mathrm{~g}$ hydrochloric acid was added to this solution under vigorous stirring. The reaction tube was sealed and the viscous gel was prehydrolyzed at $60{ }^{\circ} \mathrm{C}$ for three hours. Afterwards the tube was opened, the gel dried at room temperature for seven days and altered at $60{ }^{\circ} \mathrm{C}$ for $24 \mathrm{~h}$. The template was extracted by suspending the obtained material in a solution of $50 \%$ by mass conc. hydrochloric acid in ethanol for five days at $60{ }^{\circ} \mathrm{C}$. The material was filtered, washed with ethanol and diethyl ether and dried in an oven at $105{ }^{\circ} \mathrm{C}$ to obtain $0.24 \mathrm{~g}$ of the product as a white powder.

Polymerization of EDOT(3,4-ethylenedioxythiophene) in UKON-2a. $130 \mathrm{mg}$ UKON-2a was dried in a vacuum for several hours and homogeneously suspended in $2 \mathrm{ml}$ of a saturated solution of dry iron(III)-chloride in dry methanol $\left(20 \mathrm{mg} \mathrm{ml}^{-1}\right)$. Afterwards the solvent was evaporated very slowly $(3 \mathrm{~h})$. This procedure was repeated with $1.5 \mathrm{ml}$ of a saturated solution of dry iron(III)-chloride in dry methanol. After complete removal of the solvent, the yellow powder is cooled down to $-55{ }^{\circ} \mathrm{C}$. A solution of $30 \mathrm{mg}$ of 3,4-ethylenedioxythiophene in $0.5 \mathrm{ml}$ of chloroform was added slowly to the slightly stirred material. The material was soaked in the solution in large part. Excessive solvent was removed. The reaction mixture was warmed to room temperature and reacted for $12 \mathrm{~h}$. Afterwards the mixture is extracted with a solution of hydrochloric acid in ethanol(5\% by mass) and washed repeatedly with ethanol. The material was 
dried in a vacuum for $12 \mathrm{~h}$ and obtained as a black powder (150 mg).

Infiltration of vanadium ions into the porous host. $30 \mathrm{mg}$ of PEDOT@UKON-2a or PEDOT were suspended in $1.5 \mathrm{ml}$ of triethylamine for three hours. The material was washed with $1.5 \mathrm{ml}$ triethylamine and dried in a vacuum at $45{ }^{\circ} \mathrm{C}$ for 12 hours. $1.5 \mathrm{ml}$ of a saturated $\mathrm{VO}(\mathrm{acac})_{2}$ solution in toluene $\left(10 \mathrm{mg} \mathrm{ml}^{-1}\right.$ - the excessive $\mathrm{VO}(\mathrm{acac})_{2}$ is filtered) are added. After stirring for 72 hours at room temperature the material was extracted several times with toluene and then suspended in triethylamine for three hours. Finally the material was washed with triethylamine and diethyl ether and dried in a vacuum at $45{ }^{\circ} \mathrm{C}$ for 12 hours. For the reference materials the same conditions were used except for using a solution of acetylacetone in toluene $(11.6 \mathrm{ml} / \mathrm{ml})$ instead of a $\mathrm{VO}(\mathrm{acac})_{2} /$ toluene solution.

\section{Analytical methods}

NMR-spectra were acquired on a Bruker Avance III 400 spectrometer using $\mathrm{CDCl}_{3}$ as a solvent. Solid-state NMR spectra were recorded using a Bruker DRX 400 spectrometer. The TEM images were obtained on a Zeiss Libra 120 at $120 \mathrm{kv}$ acceleration voltage. FT-IR spectra were recorded by using a Perkin Elmer Spectrum 100 spectrometer using a ATR unit. Small-angle X-ray scattering (SAXS) measurements were conducted with a Bruker AXS Nanostar. $\mathrm{N}_{2}$-physisorptions measurements were recorded on a Micromeritics Tristar. A summary of some textural data is given in ESI S5. $\dagger$ The UV/Vis measurements were performed on a Varian Cary 100 scan UV/Vis spectrophotometer equipped with an Ulbricht reflecting sphere. Line-scan EDX data were acquired on a JEOL JEM 2200FS equipped with an EDX detector. TGA was performed on equipment from Netzsch (STA 429). SEM images and the EDX data were acquired on a Zeiss Crossbeam IS40XB instrument. Impedance was measured with a Zahner IM 6 using a 2 electrode setup with $0.3 \mathrm{~V}$ DC and $100 \mathrm{mV}$ AC. Approximately $20 \mathrm{mg}$ of material were used to press pellets with 1.5 tons. The pellet diameter was $0.8 \mathrm{~cm}$. The data were fitted/ simulated using the program ZView. Specific resistances were calculated using the formula: $\rho=R \times \pi \times d^{2} / 4 l$; with $r=$ specific resistance; $R=$ resistance; $d=$ pellet diameter; $l=$ pellet height. Specific conductivities were calculated using the formula: $\sigma=1 / \rho$. Pellet thicknesses were measured by SEM by breaking the pellets. Cyclic voltammetry measurements were performed in aqueous electrolytes $\left(c\left(\mathrm{LiClO}_{4}\right)=1 \mathrm{M}\right)$ versus a Ag/ $\mathrm{AgCl}$ reference electrode; the voltage speed rate was $50 \mathrm{mV} \mathrm{s}^{-1}$.

\section{Results and discussion}

\section{Intra-pore polymerization of PEDOT in mesoporous organosilica materials}

The first challenge that needs to be mastered is to ensure the presence of significant amounts of conducting polymer inside the pores of the PMO materials. As concrete systems for our work, we have selected PEDOT and UKON-2a (see Scheme 1).

PEDOT is like other important members from the polythiophene family insoluble in any solvent. Therefore, the infiltration of a solution containing a preformed PEDOT is excluded. Instead, it is necessary to carry out the preparation of the polymer under confined conditions inside the pores of the PMO host, which represents a particular difficulty. Our idea is that polymerization can be performed only inside the porevolume. This will also improve the contact of PEDOT with the functional groups at the surfaces of the PMO materials (Scheme 1). The described procedure (see also the Experimental part) is applicable to various thiophene derivatives (for example unmodified thiophene or 3-hexylthiophene) and also to different mesoporous materials like pure silica of the SBA-15 type and of course also for the organosilica materials of the UKON-type relevant to the current contribution. Because EDOT can be polymerized using gentle oxidation agents like $\mathrm{Fe}^{\mathrm{III}}$, iron(III)-chloride has been deposited inside the pore system (Fig. 1). $\mathrm{FeCl}_{3}$ is not a catalyst, but it reacts as a reagent. Therefore, the amount of $\mathrm{FeCl}_{3}$ inside the pores will control the degree of polymerization and the amount of polymer in the composite. At the same time it is important that the pores are still accessible for the monomer and are not blocked by solid salt particles. Therefore, at first it was necessary to characterize the $\mathrm{FeCl}_{3} @ U$ UON2a material in detail. The presence of iron chloride could be confirmed by energy dispersive X-ray spectroscopy (EDX) shown in ESI S1a. $\dagger$ One can clearly identify all expected lines FeL $\alpha(0.70 \mathrm{keV}), \mathrm{FeK} \alpha(6.4 \mathrm{keV}), \mathrm{ClK} \alpha(2.62 \mathrm{keV})$, $\mathrm{SiK} \alpha(1.74 \mathrm{keV})$, and $\mathrm{OK} \alpha(0.53 \mathrm{keV})$. A high amount of $\mathrm{FeCl}_{3}$ in the sample $(\approx 33$ mass\%) shows the success of the infiltration.

Thermogravimetric analysis (TGA) shown in Fig. 2a was applied as an independent method for quantification of the amount of $\mathrm{FeCl}_{3}$. Four major mass loss steps can be identified by means of calculation of the first derivative of the TGA data.

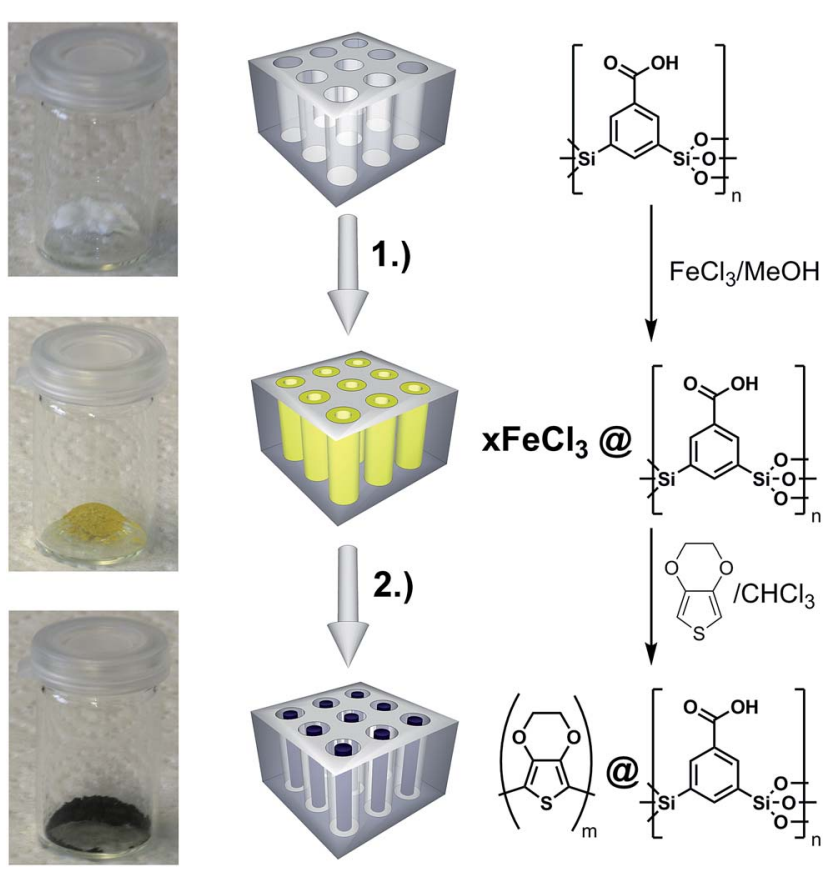

Fig. 1 Two-step synthesis of PEDOTaUKON-2a: (1) infiltration of $\mathrm{FeCl}_{3}$ into the mesopores; (2) polymerization of (3,4-ethylenedioxythiophene) inside the mesopores. 

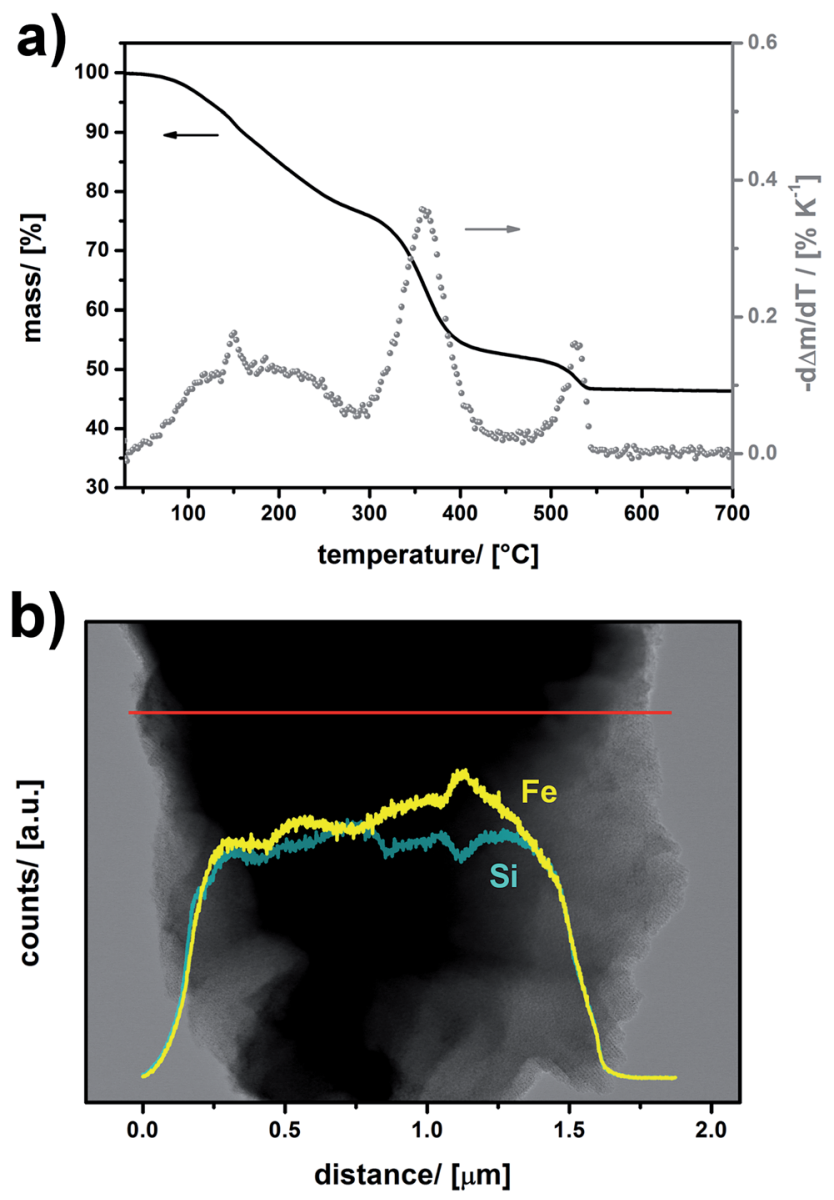

(a)

Fig. 2 (a) TGA data of $\mathrm{FeCl}_{3}$ (aUKON2a (black line) and differentiation of the TGA curve (grey dots). (b) HR-TEM EDX-line scan data of $\mathrm{FeCl}_{3} @ \cup$ KON-2a.

We assign the broad signal at $T=50-250{ }^{\circ} \mathrm{C}$ to the removal of water originating from successive polycondensation of residual $\mathrm{Si}-\mathrm{OH}$ groups at elevated temperature, to the removal of methanol coordinating to $\mathrm{FeCl}_{3}$ and/or residual isopropoxy groups from the sol-gel precursor. There is a sharp step at $T=150{ }^{\circ} \mathrm{C}$, which can be assigned to the decarboxylation benzoic acid in UKON-2a. However, the most pronounced mass loss $(\Delta m=-25 \%)$ takes place at $T_{\max }=359^{\circ} \mathrm{C}$. This correlates well with the boiling point of $\mathrm{FeCl}_{3}\left(319^{\circ} \mathrm{C}\right)$. The shift to higher temperature can easily be explained by capillary forces (Kelvin equation), and is a hint that $\mathrm{FeCl}_{3}$ is located inside the pores. The latter assumption was confirmed by transmission electron microscopy (TEM) combined with EDX line scans (Fig. 2b). One can also see from Fig. $2 \mathrm{~b}$ that the distribution of $\mathrm{Fe}^{\mathrm{III}}$ is homogeneous over the entire material. The final TGA signal (Fig. 2a) at $T=530{ }^{\circ} \mathrm{C}$ is caused by the decomposition of the remaining organic parts in UKON-2a matching to the decomposition of pure UKON-2a (Fig. 3c). TEM images of $\mathrm{FeCl}_{3}$ @UKON2a recorded at a higher magnification are shown in S1b. $\uparrow$ They prove that the immobilization of $\mathrm{FeCl}_{3}$ does not corrupt the ordered mesostructure of UKON-2a. This is also confirmed by small angle X-ray scattering analysis (SAXS) given in S1c. $\dagger$ Except for minor differences in the intensity, the SAXS
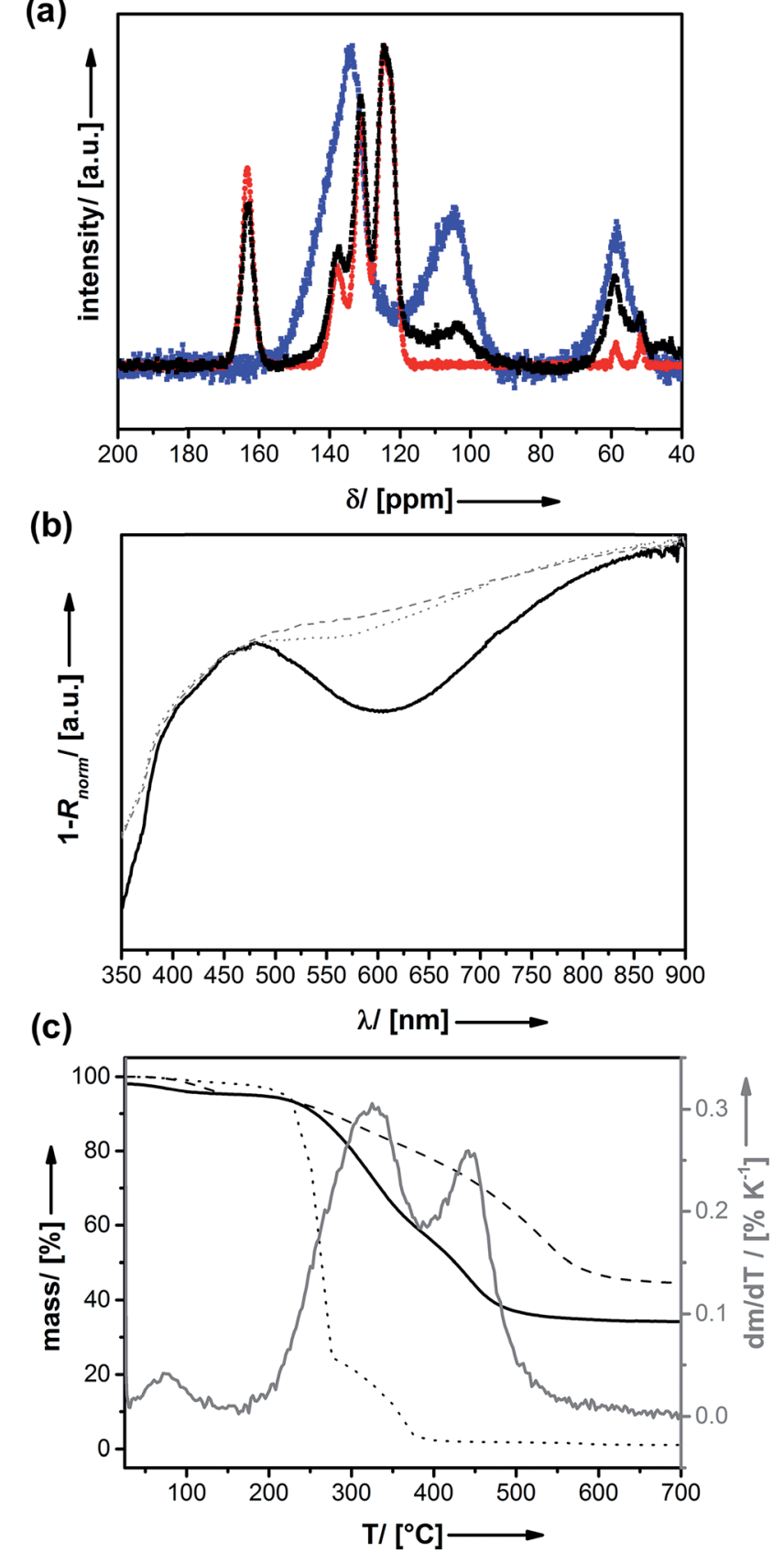

Fig. 3 (a) ${ }^{13} \mathrm{C}$ - solid-state NMR data of UKON-2a (red graph) and PEDOT (blue graph) as references and PEDOT@UKON-2a (black graph). (b) UV/Vis data of PEDOT@UKON-2a (black line), pure PEDOT (grey dotted line) and pure PEDOT obtained by dissolving PEDOT (aUKON-2a (grey dashed line). (c) TGA data of UKON-2a (black dashed line), PEDOT (black dots) as references, PEDOT(aUKON-2a (black line) and first derivative of the TGA data of PEDOT@UKON-2a (grey line).

pattern remains the same before and after $\mathrm{FeCl}_{3}$ incorporation. If there is still a certain degree of accessibility remaining, it was examined using $\mathrm{N}_{2}$ physisorption analysis shown in S1d. $\dagger$ Because a significant part of the pore volume is now filled with $\mathrm{FeCl}_{3}$ it is expected that the amount of adsorbed gas drops significantly, which can also be seen from the data. However, there seems to be still a slight porosity in the system. 
Thus, the EDOT monomer was infiltrated next. Infiltration was performed at low temperatures for prohibiting polymerization before diffusion of the monomer into the deeper parts of the porous system. Polymerization is started by increasing the temperature to $25{ }^{\circ} \mathrm{C}$. It also needs to be mentioned that, because $\mathrm{Fe}$ (III) is a reagent, the oxidative reaction with PEDOT leads to the formation of Fe(II) species. The enhanced solubility of $\mathrm{Fe}(\mathrm{II})$ species liberates space in the pores for the forming polymer. After polymerization has occurred, the residual iron salts are removed using liquid-liquid extraction. The absence of irons ions can be shown by EDX analysis (see ESI S2a $\dagger$ ). As expected, one now sees a signal for sulphur (SK $\alpha ; 2.30 \mathrm{keV})$ originating from the presence of PEDOT. The change of the colour of the material from yellow for $\mathrm{FeCl}_{3} @ U K O N-2 \mathrm{a}$ to black for PEDOT@UKON-2a is a first hint for the success of the polymerization and the formation of the conducting polymer. ${ }^{61}$ Solid-state NMR spectroscopy $\left({ }^{13} \mathrm{C}\right)$ was applied for further characterization (Fig. 3a). ${ }^{13} \mathrm{C}-\mathrm{NMR}$ data of PEDOT@UKON2a show besides the signal characteristic for the starting material UKON-2a $(\delta=124,131$, and $163 \mathrm{ppm})$ broad peaks at $\delta=103$ and $58 \mathrm{ppm}$ which can be assigned to the presence of PEDOT in accordance to the spectrum of the pure polymer measured as a reference.

The optical properties of the material were characterized using UV/Vis spectroscopy recorded in diffuse reflection shown in Fig. 3b. The absorption band at $\lambda_{1}=480 \mathrm{~nm}$ can be assigned to PEDOT in its undoped state, whereas the broad absorption signal beginning at $\lambda_{2} \approx 650 \mathrm{~nm}$ stands for electronically doped PEDOT species. ${ }^{62}$ In comparison to the optical transition $\left(\lambda_{1}=\right.$ $550 \mathrm{~nm})^{62}$ known from the literature there is a blue-shift, which might suggest that the PEDOT conjugation length is smaller, for instance because of a lower polymerization degree. ${ }^{63,64}$ To find out, if this effect is caused by the polymerization process performed under confined conditions inside the porous host, two reference experiments (A and B) were performed. (A) It is known that the Si-O-Si UKON-PMOs can be cleaved under basic conditions $(\mathrm{pH}>11){ }^{30}$ Thus, the alkaline treatment of PEDOT@UKON2a allowed us to separate PEDOT from the mesoporous host. UV/Vis data for this PEDOT are compared in Fig. 3 to a PEDOT polymer prepared by polymerization in bulk solution (B) under otherwise unvarying conditions. The two spectra are almost identical, indicating that the polymerization in pores does not significantly alter the chemical nature of the polymer, for instance the polymerization degree. However, it can be seen that the porous host has the effect that the absorption signals in PEDOT@UKON2a are much more distinct. This might be explained by a difference in chain conformation of PEDOT inside the pores. ${ }^{65,66}$ The existence of the conducting polymer as well as first hints for particular electronic properties of the PEDOT@UKON2a composite can also be seen from scanning electron microscopy (SEM) investigation (given in $\mathrm{S} 2 \mathrm{~b} \dagger$ ). The morphologies of the empty UKON-2a and the PEDOT filled variant look similar. The particles of the mesoporous solid show flat surfaces without any polymeric residues on or between those particles. Polymerization seems to have occurred only inside the pores. One marked difference is, however, that the particles containing PEDOT display much weaker charging effects in the electron beam, which is a direct result of its conducting nature. As an independent technique, it was checked by atomic force microscopy (AFM), if significant soft zones are present in the material (see S2d $\dagger$ ). This would show that a significant amount of polymer is found outside the particles. However, the latter is not the case.

Quantification of the amount of PEDOT is not yet possible by means of the mentioned analytical techniques. The exact amount of polymer in the nanocomposite can be determined using TGA (Fig. 3c). There are two main mass-loss steps. One at $T=468{ }^{\circ} \mathrm{C}$, which can be assigned to the oxidative removal of the organic part of the PMO matrix $\left(\mathrm{C}_{6} \mathrm{H}_{3} \mathrm{R}\right)$, and one at $340{ }^{\circ} \mathrm{C}$ which is caused by the removal of PEDOT. Comparison to the TGA trace of pure PEDOT (Fig. $3 \mathrm{c} ; T_{\text {Dec }}=252^{\circ} \mathrm{C}$ ) indicated that its stability is increased inside the mesoporous matrix, possibly because of confinement effects. ${ }^{26}$ The quantitative evaluation of the mentioned, two steps shows that the material is composed of $\approx 23$ mass\% PEDOT (equates to $32 \mathrm{~mol} \%$ PEDOT). EDX was used as an independent technique for confirming the TGA results (see S2a $\dagger$ ). The amount of sulphur present indicates that the material consists of $34 \mathrm{~mol} \%$ PEDOT, which is in excellent agreement with the TGA data. Please note that this represents a very high loading of the pores with the organic polymer. ${ }^{50,67,68}$ As already stated above other procedures can yield comparable amounts of polymer or even more but with significant amounts of polymer outside the pores. ${ }^{53-55}$ Our materials can also be loaded with lower amounts of polymer. Because Fe(III) is a reagent needed for polymerization, the deposition of a smaller amount of $\mathrm{FeCl}_{3}$ inside the pores and adjustment of the amount of monomer will lead to a drop of the polymer content. However, because we were focusing on maximizing the polymer content, we were not following this point further.

It is important to check, if the pores of the PMO are still accessible or not and how the polymer is distributed. The samples were investigated by $\mathrm{N}_{2}$ physisorption analysis before and after polymerization of PEDOT inside the pore-system (Fig. 4a). Because the space occupied by PEDOT is no longer accessible for nitrogen molecules, it is clear that the porevolume decreases. Considering 23 mass\% PEDOT (see above) in the composite and the density of PEDOT $\left(\rho=1.46 \mathrm{~g} \mathrm{~cm}^{-3}\right)$, one expects a decrease of the pore-volume $\left(\Delta V_{\text {pore }}\right)$ of $0.22 \mathrm{~cm}^{3} \mathrm{~g}^{-1}$. This is a little bit lower than the value of $\Delta V_{\text {pore }}=0.33 \mathrm{~cm}^{3} \mathrm{~g}^{-1}$ calculated from the experimental pore volumes of UKON-2a $\left(0.79 \mathrm{~cm}^{3} \mathrm{~g}^{-1}\right)$ compared with PEDOT@UKON2a $\left(0.45 \mathrm{~cm}^{3} \mathrm{~g}^{-1}\right)$. The specific surface area decreases only a bit from $694 \mathrm{~m}^{2} \mathrm{~g}^{-1}$ for the host material to $541 \mathrm{~m}^{2} \mathrm{~g}^{-1}$ for PEDOT@UKON2a. The latter findings suggest that the polymer is not blocking the pores, but it is homogeneously coating the pore-walls. This assumption is supported by the fact that the Barret-JoynerHalenda $(\mathrm{BJH})$ pore-size distribution function, calculated by the adsorption branches of the isotherms, of PEDOT@UKON-2a (Fig. 4a) is shifted slightly towards smaller values $\left(\Delta D_{\text {pore }}=\right.$ $0.45 \mathrm{~nm}$ ). A similar effect can also be seen in TEM (Fig. 4c and d) $\left(\Delta D_{\text {pore }} \approx 0.5 \mathrm{~nm}\right)$. Thus the change in pore-size is because of a PEDOT coating of the pore-surfaces and not of a change in overall periodicity, could be confirmed by SAXS measurements shown in S2c, $\dagger$ because the main signal at $q_{10}=0.55 \mathrm{~nm}^{-1}$ is 

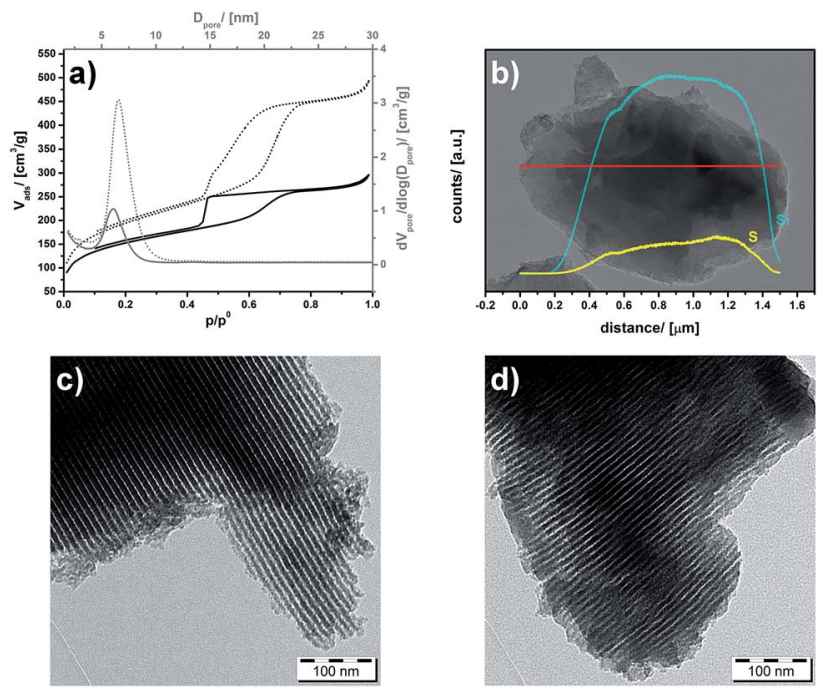

Fig. 4 (a) $\mathrm{N}_{2}$ physisorption data (black) and BJH pore-size distribution function (grey) of UKON-2a (dotted curves) and PEDOTAUKON-2a (solid curves). (b) HR-TEM EDX-linescan data of PEDOTAUKON-2a. (c) TEM micrograph of UKON-2a. (d) TEM micrograph of PEDOT@UKON-2a.

not shifted. Additionally, an HR-TEM EDX line scan shows that the polymer is distributed homogenously over the material and not only on the pore entrances (Fig. 4b). The same result was obtained from HRTEM-EDX line scan measurements given in S2e. $\dagger$ This leads to the conclusion that a material has been gained, which not only has a huge interface area between two functional materials, but also still retains its large surface area and the accessibility of the pores (as depicted in Scheme 1).

\section{Investigation of electronic and stimuli-responsive properties}

The next step is to examine the responsive behaviour of the materials. From our previous experiences with UKON-2a we know that the carboxylic groups are suitable for the coordination to oxophilic transition metal species like the vanadyl cation $[\mathrm{VO}]^{2+} \cdot{ }^{\mathbf{2 8 , 6 9}}$ Therefore, vanadyl acetylacetonate $\left([\mathrm{VO}](\mathrm{acac})_{2}\right)$ was infiltrated into PEDOT@UKON2a resulting in a sample labelled as VO@PEDOTUKON2a. From EDX data (given in $\mathrm{S} 3 \dagger$ ) it can be concluded that VO@PEDOTUKON2a contains $27 \%[\mathrm{VO}]^{2+}$ ions per PEDOT monomer unit. Because $[\mathrm{VO}]^{2+}$ itself is colourless, inspection of the optical properties of VO@PEDOTUKON2a (Fig. 5) can be used as a first tool to investigate any effects on the electronic properties of the conducting polymer. It can be seen that the absorption band corresponding to the doped state of PEDOT $(\lambda>700 \mathrm{~nm})$ has gained intensity. This indicates that $[\mathrm{VO}]^{2+}$ as a strong Lewisacid also coordinates to PEDOT (as depicted in Scheme 1) and presumably leads to a p-doped character. An analogous procedure was performed for the pure polymer as a reference (see S4 $\dagger$ ). The treatment with [VO](acac) $)_{2}$ had no effect on the optical properties of pure PEDOT, and this is a first hint for the importance of the presence of the functional groups in the PMO material.
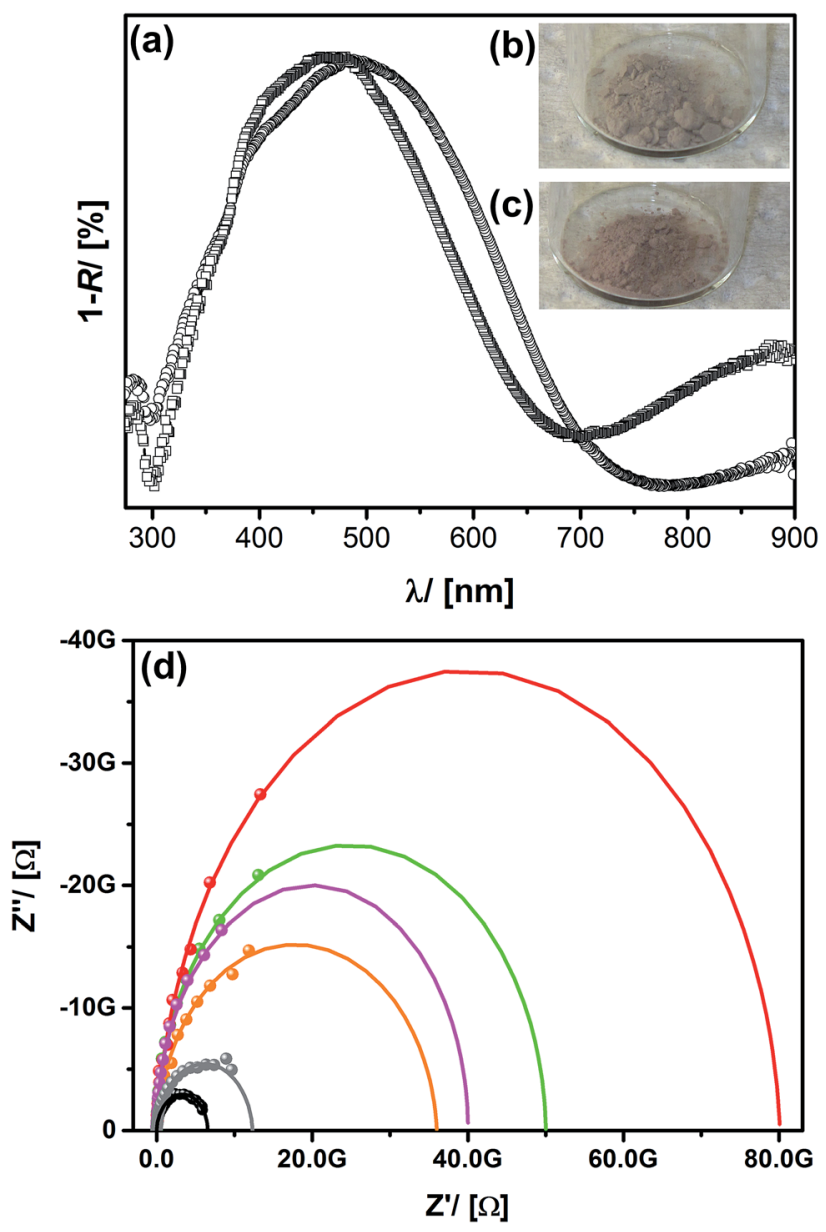

Fig. 5 (a) UV/Vis diffuse reflectance $(R)$ data of VO@PEDOTUKON2a (squares) and PEDOTCUKON-2a as a reference (circles). Intensities were normalized to the absorption band at $\lambda=480 \mathrm{~nm}$. (b and c) Photographs of PEDOT(aUKON-2a (b) with [VO] ${ }^{2+}$ ions (c) reference without $[\mathrm{VO}]^{2+}$. (d) Impedance data (data points) and fitted curves (solid lines); Nyquist plots with $Z^{\prime} \cong$ real part of impedence and $Z^{\prime \prime}=$ imaginary part of impedence; black $\cong$ VO@PEDOTUKON2a; grey $\cong$ PEDOTAUKON2a; orange $\cong$ FeaPEDOTUKON2a; blue $\cong N i @ P E D O T U K O N 2 a$; green $\cong$ Co@PEDOTUKON2a; red $\cong$ Mn@PEDOTUKON2a.

If it is the case that promoted p-doping has occurred for VO@PEDOTUNKON2a, one has to expect a change in conductivity compared to PEDOT@UKON2a. Therefore, the samples were examined using impedance spectroscopy (see Fig. 5d; Experimental part). Indeed the resistivity decreases from $6.8 \mathrm{G} \Omega$ for PEDOT@UKON2a to $4.3 \mathrm{G} \Omega$ for VO@PEDOTUNKON2a. Alternative cations like $\mathrm{Fe}^{2+}, \mathrm{Ni}^{2+}$, $\mathrm{Co}^{2+}$ or $\mathrm{Mn}^{2+}$ have also been tested, and interestingly every species leads to different conductivities (Fig. 5d). The latter results lead the way towards developing a potential sensor for transition metal containing solutions. However, it should also be noted that weaker Lewis-acids have an opposite effect on the electronic properties of the PEDOT@UKON2a hybrid compared to the strong Lewis-acid $[\mathrm{VO}]^{2+}$, as they lead to a decrease in conductivity. It seems that weak Lewis-acids shift the equilibrium to the undoped state of PEDOT. 
Further evidence for the electronic contact between transition metal cations coordinated to the carboxyl groups in $\mathrm{UKON}-2 \mathrm{a}$ and the conducting polymer inside the pores comes from cyclic voltammetry measurements (CV). Ru@PEDOTUKON2a was prepared and investigated using $\mathrm{CV}$ in comparison to PEDOT@UKON2a (Fig. 6). The CV curve of PEDOT@UKON2a is characterized by two broad waves, one for oxidation and another for reduction of PEDOT. Because of a potential overlap with these signals a precise evaluation of the $\mathrm{CV}$ data of Ru@PEDOTUKON2a is difficult. However, one can clearly see additional signals for the $\mathrm{Ru}^{\mathrm{II}} / \mathrm{Ru}^{\mathrm{III}}$ redox pair. Because $\mathrm{Ru}$ containing materials are used for pseudo-capacitive energy storage and electrocatalysis, ${ }^{\mathbf{7 0 , 7 1}}$ the latter results are important for further potential applications of the materials presented in the current paper. It is also seen that current $I$ increases by a factor of 20 for Ru@PEDOTUKON2a in comparison to PEDOT@UKON2a (Fig. 6). This shows that the interplay between the three components UKON-2a, PEDOT and the transition metal species (see also Scheme 1) is a deciding factor for the amount of charges that can be stored inside the material and also regarding its electrochemical activity. The stability of PEDOT@UKON2a was checked by running $100 \times$ successive CV cycles, indicating no significant loss of the signal. In addition, FT-IR data of the material before and after these 100 cycles were compared to each other. Also in IR, there are no changes seen. The latter data are shown in ESI S6. $\dagger$

The enormous sensitivity of the PEDOT@UKON2a hybrid material against Lewis-acids can also be demonstrated by studying the $\mathrm{pH}$-dependency of the system. The material was treated with aqueous solutions with $\mathrm{pH}=0,2$, and 7. Afterwards impedance spectra were recorded (Fig. 7). One sees that also protons are capable of p-doping PEDOT inside the PMO material. The higher the proton concentration is, the lower is the electric resistance. However, these results also mean that one has to control the $\mathrm{pH}$-value carefully (which we did), when one is studying the interaction with other Lewis-acids like the mentioned transition metal species.

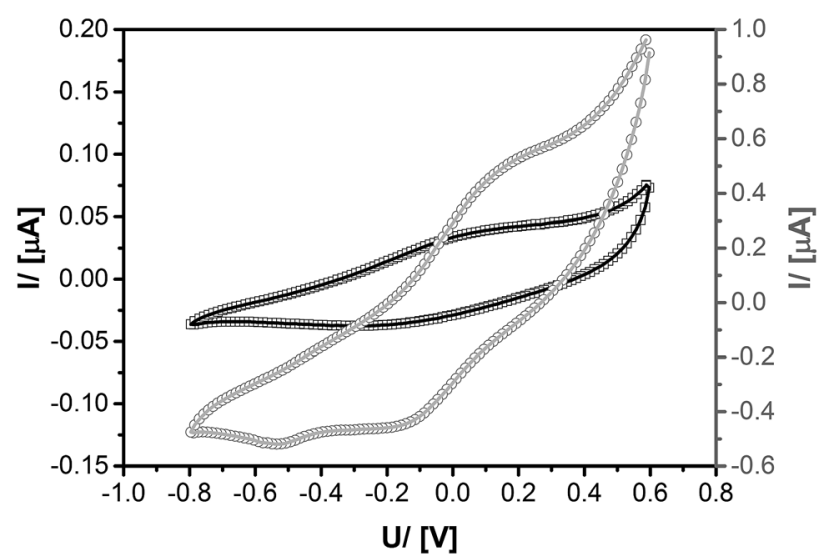

Fig. $6 \mathrm{CV}$ data of PEDOT(aUKON2a (black) in comparison to RuAPEDOTUKON2a (grey).

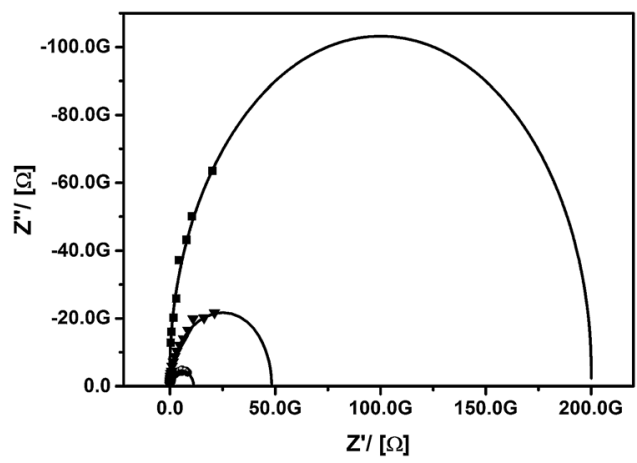

Fig. 7 Impedance spectra (experimental data points and fit curve) for PEDOT@UKON2a treated at $\mathrm{pH}=0$ (dots), 2 (triangles) and 7 (squares).

\section{Conclusions}

We have presented the successful combination of two different classes of functional materials: periodically ordered mesoporous organosilica materials and conducting polymers. First, the intra-pore polymerization of thiophene-based polymers, for example PEDOT, was mastered. The advantage is that a large amount of polymer can be brought into contact with the large number of functional surface groups located in the pores of the PMO material. The potential of those functional groups was used for the coordination of different Lewis-acids, e.g. transition metal cations. It could be shown that an electronic contact between those transition metal species and PEDOT is realized. Furthermore, the different Lewis-acids led to specific changes in the doping state of PEDOT. The strength of the presented materials is presumably not due to the realization of a very low detection limit, which is not determined, but the possibility to differentiate between the different transition metal species using only one sensory system. The mentioned properties make the materials not only interesting as smart materials, but also for applications especially in sensor arrays, electrocatalysis or even as supercapacitors.

\section{Acknowledgements}

We thank the University of Konstanz for funding.

\section{Notes and references}

1 C. M. Friend, Interdiscip. Sci. Rev., 1996, 21, 195-198.

2 F. Garnier, Angew. Chem., Int. Ed., 1989, 28, 513-517.

3 E. Ruiz-Hitzky, Chem. Rec., 2003, 3, 88-100.

4 W. B. Spillman, J. S. Sirkis and P. T. Gardiner, Smart Mater. Struct., 1996, 5, 247-254.

5 M. A. C. Stuart, W. T. S. Huck, J. Genzer, M. Muller, C. Ober, M. Stamm, G. B. Sukhorukov, I. Szleifer, V. V. Tsukruk, M. Urban, F. Winnik, S. Zauscher, I. Luzinov and S. Minko, Nat. Mater., 2010, 9, 101-113.

6 F. Xia and L. Jiang, Adv. Mater., 2008, 20, 2842-2858.

7 C. D. H. Alarcon, S. Pennadam and C. Alexander, Chem. Soc. Rev., 2005, 34, 276-285. 
8 C. Bechinger, S. Ferrere, A. Zaban, J. Sprague and B. A. Gregg, Nature, 1996, 383, 608-610.

9 S. Polarz and B. Smarsly, J. Nanosci. Nanotechnol., 2003, 2, 581.

10 F. Hoffmann, M. Cornelius, J. Morell and M. Froeba, Angew. Chem., Int. Ed., 2006, 45, 3216-3251.

11 A. Thomas, Angew. Chem., Int. Ed., 2010, 49, 8328-8344.

12 S. S. Park, M. S. Moorthy and C. S. Ha, NPG Asia Mater., 2014, 6, e96.

13 F. Hoffmann and M. Froeba, Chem. Soc. Rev., 2011, 40, 608620.

14 W. D. Wang, J. E. Lofgreen and G. A. Ozin, Small, 2010, 6, 2634-2642.

15 G. L. Athens, R. M. Shayib and B. F. Chmelka, Curr. Opin. Colloid Interface Sci., 2009, 14, 281-292.

16 N. Mizoshita, T. Tani and S. Inagaki, Chem. Soc. Rev., 2011, 40, 789-800.

17 W. Wang, J. E. Lofgreen and G. A. Ozin, Small, 2010, 6, 26342642.

18 P. Van Der Voort, D. Esquivel, E. De Canck, F. Goethals, I. Van Driessche and F. J. Romero-Salguero, Chem. Soc. Rev., 2013, 42, 3913-3955.

19 T. Asefa, M. J. MacLachan, N. Coombs and G. A. Ozin, Nature, 1999, 402, 867-871.

20 S. Inagaki, S. Guan, Y. Fukushima, T. Ohsuna and O. Terasaki, J. Am. Chem. Soc., 1999, 121, 9611-9614.

21 B. J. Melde, B. T. Holland, C. F. Blanford and A. Stein, Chem. Mater., 1999, 11, 3302-3308.

22 S. S. Park, M. S. Moorthy and C. S. Ha, NPG Asia Mater., 2014, 6, 21.

23 S. Inagaki, S. Guan, T. Ohsuna and O. Terasaki, Nature, 2002, 416, 304-307.

24 A. Kuschel and S. Polarz, Adv. Funct. Mater., 2008, 18, 12721280.

25 A. Kuschel, H. Sievers and S. Polarz, Angew. Chem., Int. Ed., 2008, 47, 9513-9517.

26 S. Polarz and A. Kuschel, Chem.-Eur. J., 2008, 14, 9816-9829.

27 A. Kuschel, M. Drescher, T. Kuschel and S. Polarz, Chem. Mater., 2010, 22, 1472.

28 A. Kuschel, M. Luka, M. Wessig, M. Drescher, M. Fonin, G. Kiliani and S. Polarz, Adv. Funct. Mater., 2010, 20, 11331143.

29 A. Kuschel and S. Polarz, J. Am. Chem. Soc., 2010, 132, 65586565.

30 M. Luka and S. Polarz, Microporous Mesoporous Mater., 2013, 171, 35-43.

31 M. Wessig, M. Drescher and S. Polarz, J. Phys. Chem. C, 2013, 117, 2805-2816.

32 J. Gehring, D. Schleheck, M. Luka and S. Polarz, Adv. Funct. Mater., 2014, 24, 1140-1150.

33 S. Mascotto, D. Wallacher, A. Kuschel, S. Polarz, G. A. Zickler, A. Timmann and B. M. Smarsly, Langmuir, 2010, 26, 65836592.

34 C. J. Brabec, N. S. Sariciftci and J. C. Hummelen, Adv. Funct. Mater., 2001, 11, 15-26.

35 J. Roncali, Chem. Rev., 1992, 92, 711-738.
36 B. L. Groenendaal, F. Jonas, D. Freitag, H. Pielartzik and J. R. Reynolds, Adv. Mater., 2000, 12, 481-494.

37 I. F. Perepichka, D. F. Perepichka, H. Meng and F. Wudl, Adv. Mater., 2005, 17, 2281-2305.

38 M. Gerard, A. Chaubey and B. D. Malhotra, Biosens. Bioelectron., 2002, 17, 345-359.

39 J. Janata and M. Josowicz, Nat. Mater., 2003, 2, 19-24.

40 P. Bauerle and S. Scheib, Adv. Mater., 1993, 5, 848-853.

41 U. Lange, N. V. Roznyatouskaya and V. M. Mirsky, Anal. Chim. Acta, 2008, 614, 1-26.

42 E. Bundgaard and F. C. Krebs, Sol. Energy Mater. Sol. Cells, 2007, 91, 954-985.

43 V. Ganesan and A. Walcarius, Langmuir, 2004, 20, 36323640 .

44 Q. L. Wang, G. X. Lu and B. J. Yang, Langmuir, 2004, 20, 1342-1347.

45 A. Walcarius, D. Mandler, J. A. Cox, M. Collinson and O. Lev, J. Mater. Chem., 2005, 15, 3663-3689.

46 P. Gomez-Romero, Adv. Mater., 2001, 13, 163-174.

47 S. Kirchmeyer and K. Reuter, J. Mater. Chem., 2005, 15, 20772088.

48 C. G. Wu and T. Bein, Science, 1994, 264, 1757-1759.

49 C. G. Wu and T. Bein, Chem. Mater., 1994, 6, 1109-1112.

50 R. Silva and T. Asefa, Adv. Mater., 2012, 24, 1878-1883.

51 V. S. Y. Lin, D. R. Radu, M. K. Han, W. H. Deng, S. Kuroki, B. H. Shanks and M. Pruski, J. Am. Chem. Soc., 2002, 124, 9040-9041.

52 Q. L. Cheng, V. Pavlinek, A. Lengalova, C. Z. Li, Y. He and P. Saha, Microporous Mesoporous Mater., 2006, 93, 263-269.

53 T. L. Kelly, K. Yano and M. O. Wolf, Langmuir, 2010, 26, 421431.

54 T. L. Kelly, S. P. Y. Che, Y. Yamada, K. Yano and M. O. Wolf, Langmuir, 2008, 24, 9809-9815.

55 T. L. Kelly, Y. Yamada, S. P. Y. Che, K. Yano and M. O. Wolf, Adv. Mater., 2008, 20, 2616-2621.

56 M. Tiemann, Chem.-Eur. J., 2007, 13, 8376-8388.

57 D. T. McQuade, A. E. Pullen and T. M. Swager, Chem. Rev., 2000, 100, 2537-2574.

58 S. W. Thomas, G. D. Joly and T. M. Swager, Chem. Rev., 2007, 107, 1339-1386.

59 J. Liu, G. Z. Cao, Z. G. Yang, D. H. Wang, D. Dubois, X. D. Zhou, G. L. Graff, L. R. Pederson and J. G. Zhang, ChemSusChem, 2008, 1, 676-697.

60 M. Mastragostino, C. Arbizzani and F. Soavi, J. Power Sources, 2001, 97-8, 812-815.

61 F. Jonas and L. Schrader, Synth. Met., 1991, 41, 831-836.

62 C. Kvarnstrom, H. Neugebauer, S. Blomquist, H. J. Ahonen, J. Kankare and A. Ivaska, Electrochim. Acta, 1999, 44, 27392750.

63 F. Tran-Van, S. Garreau, G. Louarn, G. Froyer and C. Chevrot, J. Mater. Chem., 2001, 11, 1378-1382.

64 F. Tran-Van, S. Garreau, G. Louarn, G. Froyer and C. Chevrot, Synth. Met., 2001, 119, 381-382.

65 A. L. Botelho, Y. Shin, J. K. Liu and X. Lin, PLoS One, 2014, 9, 8. 
66 A. P. Monkman, L. O. Palsson, R. W. T. Higgins, C. S. Wang, M. R. Bryce, A. S. Batsanov and J. A. K. Howard, J. Am. Chem. Soc., 2002, 124, 6049-6055.

67 F. Cucinotta, F. Carniato, G. Paul, S. Bracco, C. Bisio, S. Caldarelli and L. Marchese, Chem. Mater., 2011, 23, 2803-2809.

68 M. S. Cho, H. J. Choi and W. S. Ahn, Langmuir, 2004, 20, 202207.
69 A. Kuschel, M. Drescher, T. Kuschel and S. Polarz, Chem. Mater., 2010, 22, 1472-1482.

70 G. P. Wang, L. Zhang and J. J. Zhang, Chem. Soc. Rev., 2012, 41, 797-828.

71 J. S. Spendelow and A. Wieckowski, Phys. Chem. Chem. Phys., 2007, 9, 2654-2675.

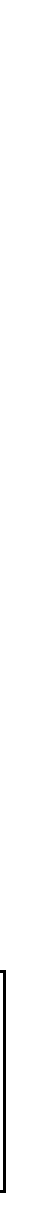

